Revue d'histoire de l'Amérique française

RAS REVUE D.HISTOIRE DE L'AMÉRIQUE FRANÇAISE

\title{
Pour une épistémè ouverte, plurielle et compréhensive
}

\section{Jocelyn Létourneau}

Volume 63, numéro 1, été 2009

URI : https://id.erudit.org/iderudit/039889ar

DOI : https://doi.org/10.7202/039889ar

Aller au sommaire du numéro

Éditeur(s)

Institut d'histoire de l'Amérique française

ISSN

0035-2357 (imprimé)

1492-1383 (numérique)

Découvrir la revue

Citer cet article

Létourneau, J. (2009). Pour une épistémè ouverte, plurielle et compréhensive. Revue d'histoire de l'Amérique française, 63(1), 125-133.

https://doi.org/10.7202/039889ar d'utilisation que vous pouvez consulter en ligne.

https://apropos.erudit.org/fr/usagers/politique-dutilisation/ 


\section{POUR UNE ÉPISTÉMÈ OUVERTE, PLURIELLE ET COMPRÉHENSIVE'}

Je me souviens d'avoir tenu, au début des années 1980, à l'endroit de Jean Hamelin qui incarnait dans sa conception et sa pratique de l'histoire toutes les tendances qui m'irritaient chez les disciples de Clio, des propos fort critiques, presque insolents. Faisant preuve de fronde plus que de profondeur et affichant dans mon dire de la jeunesse plus que de la sagesse, je reprochais à Hamelin et à sa collègue Nicole Gagnon, qui venaient de commettre un petit ouvrage intitulé L'Homme historien ${ }^{2}$, de se complaire dans des visions dépassées et inacceptables de l'histoire ${ }^{3}$. J'en avais particulièrement contre le point de vue humaniste ou humanisant défendu par les deux professeurs qui prenaient le parti du subjectivisme contre l'objectivisme, qui récusaient les théories trop précises (en qualifiant implicitement le matérialisme historique de calamité) et qui ne craignaient pas de définir l'histoire comme étant aussi un art! Fort de mes convictions sur ce qu'était la discipline historique (combien d'heures à discuter avec Jean-Marie Fecteau, alors camarade), je m'insurgeais contre tout ce qui pouvait faire de l'histoire autre chose qu'une science positive de l'agir humain se déployant dans des rapports de classe qui déterminaient et expliquaient tout. Que des sphères de socialité restent inaccessibles à la raison cartésienne, que les mots ne puissent toujours dire ou rendre les choses et que l'ambiguité soit au cœur de la condition humaine, voilà trois postulats épistémologiques qui m'étaient insupportables. Pour contrer la mollesse analytique défendue par Hamelin et Gagnon, il fallait, croyais-je fermement, aborder l'histoire comme un corps structuré qui se prêtait à l'étude systématique et que l'on pouvait comprendre comme un tout organisé. Dans cette entreprise de réconciliation de la démarche historienne avec la (ma?) méthode scientifique, la conceptualisation stricte et la modélisation géométrique me paraissaient des outils indispensables. "Critique de l'indétermination historique, de l'approximation méthodologique et de l'hésitation historienne», tel aurait pu être le sous-titre de mon article.

1. C'est à l'invitation appuyée de la RHAF que ce texte a été rédigé.

2. Jean Hamelin et Nicole Gagnon, avec la participation d'André Garon et al., L'homme historien: introduction à la méthodologie de l'histoire (Saint-Hyacinthe/Paris, Edisem/Maloine, 1979).

3. Jocelyn Létourneau, «L'homme historien: entre l’idéalisme et le subjectivisme», Implications, 2,1 (hiver 1981): 41-53. 
À trente ans de distance, cet intitulé pourrait coiffer le texte de Thierry Nootens. On sent en effet, chez ce professeur de l'UQTR, une insatisfaction vive devant les concepts mous qui cherchent à épouser la matière du passé plutôt qu'à la conformer aux attentes et prescriptions des grands schèmes explicatifs. Nostalgique d'une époque où dominaient les lectures matérialistes du social et où, pour beaucoup d'historiens dont il est apparemment l'héritier intellectuel, les méta-structures, processus institutionnels et grands ensembles faisaient foi de tout, Nootens s'en prend notamment au concept d'identité qu'il trouve vaseux, imprécis, inefficace et, sauf exception, inutile.

Que dire de la croisade du collègue contre l'"approche identitaire»? Qu'il n'a pas tort de signaler certains excès commis par les aficionados de la "posture analytique » qui l'indispose, mais qu'il exagère dans les immodérations qu'il dénonce.

Ainsi, on ne le chicanera pas de ce qu'il affirme à propos de l'application ample du concept d'identité par des cohortes de chercheurs qui, désireux d'être dans le vent et de penser dans le cadre de l'un des paradigmes de notre temps, en ont usé et abusé jusqu'à plus soif. Ai-je moi-même fait preuve de démesure dans l'emploi du terme? Si M. Nootens le croit $^{4} \ldots$

À ma décharge - et à la décharge de mes collègues du CÉLAT durant les années 1980 et 1990 -, je soutiendrai cependant que nous avons, grâce au concept d'identité et aux problématiques dont il était le centre, ajouté aux lectures possibles de l'expérience historique québécoise. On pourrait dire des travaux menés au CÉLAT, à partir du moment où Jacques Mathieu a pris la direction du Centre au début des années 1980, qu'ils ont été pionniers dans l'exploration de domaines alors ignorés ou négligés par l'historiographie québécoise: l'étude des mémoires collectives et des espaces identitaires, celle des rapports entre mémoire, histoire et identité, celle des constructions identitaires et des grands récits collectifs, celle du patrimoine historial fondant la culture franco-québécoise, celle de la formation des grandes représentations collectives, y compris les imaginaires sociaux, et j'en passe $e^{5}$. Ces travaux ont eu de l'écho à l'étranger, notam-

4. Curieux tout de même que le collègue, pour «labelliser» ma pensée sur la question de l'identité (p. 44 de son article), utilise une citation provenant d'un texte publié début 1994, présentation d'un ouvrage collectif découlant d'un colloque et consistant en une interrogation générale sur les tenants et aboutissants de la rencontre savante organisée sous les auspices de la CÉFAN. On a déjà vu exégèse plus minutieuse...

5. Pour une présentation des axes de recherche, problématiques et travaux menés au CÉLAT dans les années 1990, voir Jocelyn Létourneau et Laurier Turgeon, «Formation et mouvance 
ment en France ${ }^{6}$. Ils ont paru dans d'importantes revues. Ils ont influencé les meilleurs historiens québécois et inspiré des centaines d'étudiants. Dans les années 1990, le département d'histoire de l'Université Laval, grâce en particulier aux problématiques développées au CÉLAT et aux concepts défrichés par ses chercheurs, est devenu un lieu de référence - au Québec, au Canada et dans le monde de la francophonie - pour l'étude de l'histoire sous l'angle de tout ce que $\mathrm{M}$. Nootens considère comme étant des objets embrouillés ou vaporeux: le rôle des discours sociaux dans la production de la société; la capacité des acteurs à déjouer ou à négocier, par des stratégies subjectives de positionnement social, les systèmes régulateurs et les logiques massificatrices; la construction des repères collectifs, aussi appelés balises identitaires; la formation de la conscience historique chez les membres d'une collectivité; etc.

Vers la même époque, le CÉLAT était également à l'origine de la critique de quelques-uns des mythistoires ayant servi de support à la formation de ce qu'on appellera, à la suite de Fernand Dumont, la référence (franco-) québécoise. Les travaux de Serge Courville et de son équipe, ceux de Laurier Turgeon et la sienne, les miens et ceux d'autres collègues ont en effet ébranlé certaines visions d'histoire cardinales à cette référence. Pensons seulement aux idées de société retardée et de nation isolée, à celles de survivance collective et de parcours déphasé ou à celles de grande noirceur et de révolution tranquille, toutes métaphores centrales dans la mémoire collective québécoise et la théogonie nationale. Sauf à faire abstraction de travaux influents, il n'est assurément plus possible de conjuguer l'expérience historique québécoise au temps de ces mythistoires ${ }^{7}$.

Qu'on me comprenne bien: je ne dis pas que les travaux réalisés au CÉLAT sont sans reproche; je n'affirme pas non plus qu'aucune innovation historienne n'a germé ailleurs que dans ce centre de recherche. Penser ainsi serait absurde. Je soutiens seulement que les travaux menés au CÉLAT ont élargi le territoire existant de l'histoire du Québec, celui qui,

contemporaine des espaces identitaires", Cahiers d'histoire du Québec au xx siècle, 4 (été 1995): 121-136.

6. Est symptomatique de l'intérêt porté, dans l'Hexagone, aux travaux menés au CÉLAT, le fait que la revue Genèses, alors montante dans le champ historien en France - et plus généralement en Europe - consacrait un article entier aux travaux des chercheurs du Centre. Voir [Gérard Noiriel], «Le CÉLAT, au cœur de la nouvelle recherche québécoise. Entretien avec Jocelyn Létourneau ", Genèses, 12 (mai 1993): 89-95.

7. À ce sujet, on s'étonne du fait que, dans son bilan de l'historiographie portant sur le Québec durant les années 1985-2002, Joanne Burgess n’ait mentionné aucune étude émanant du CÉLAT! Voir J. Burgess, «L'histoire du Québec: tendances récentes et enjeux», dans Denise Lemieux dir., et al., Traité de la culture (Québec, Les Presses de l’Université Laval, 2002), 29-45. 
dans les années 1970, avait été fort heureusement réinvesti par plusieurs historiens de l'Université du Québec à Montréal, davantage intéressés par la place des déterminations structurelles, le jeu des grands acteurs institués et le poids des morphologies structurales dans l'organisation des sociétés.

Peut-il y avoir de la place au Québec pour différentes lectures de l'histoire, qui ont chacune leurs avantages et leurs limites, mais qui sont surtout complémentaires les unes des autres? Il faut redouter le jour où un tribunal d'historiens, pour le «bien-être» de la discipline, cela va de soi, sanctionnera quelque loi de clarté historienne...

Évidemment, M. Nootens s'en prend moins aux objets d'histoire qu'aux méthodes et concepts employés pour aborder ces objets. Outre de rappeler que tout travail exploratoire est inévitablement grossier et imparfait $^{8}$, il faut comprendre que plusieurs chercheurs du CÉLAT, dont j'étais, manifestaient de l'intérêt pour les complications, les incongruités, les ambiguïtés, les diversités et les pluralités de l'histoire, plus que pour ses régularités, ses conformités, ses assiduités, ses continuités et ses unités tous terrains de prédilection du collègue. Or, mettre l'accent sur l'acteur, les représentations sociales, les constructions de sens et les productions textuelles ou langagières au sein d'une société ne signifie pas de rejeter le reste. Il s'agit de reconnaître que la société peut être envisagée simultanément "par le haut» et "par le bas», du point de vue de ses régulations macroscopiques et de ses singularités microscopiques; que l'être humain, tout en participant d'une société orchestrée ou contrôlée par des pouvoirs et s'inscrivant dans des configurations particulières de rapports sociaux, ne perd jamais la capacité de produire du sens qui lui est privé, sens qui constitue aussi le socle de sa subjectivité et de son identité ; et que le discours n'est pas extérieur à la vie, mais constitutif de l'agir humain et de l'expérience du monde, ce qui fait de la réalité humaine un vécu tout autant qu'un construit.

Dans ces positions épistémologiques, $\mathrm{M}$. Nootens voit beaucoup de mauvais. À ses yeux, le courant dans lequel je m'inscris (d'après lui) n'en aurait que pour l'étude de la société comme abstraction culturelle ou

8. J'ai présenté mes points de vue sur l'exploration savante comme démarche de recherche dans Jocelyn Létourneau, «Bogumil Jewsiewicki, ou l'histoire comme exploration savante», dans Isidore Ndaywel è Nziem et Élizabeth Mudimbe-Boyi, dir., Une histoire en partage : images, mémoires et savoirs. Mélanges offerts à Bogumil Jewsiewicki (Paris, Khartala, 2009), 11-19. 
textuelle et délaisserait la prise en compte du "social concret» ; l'individu étant le point de départ et d'arrivée de "nos» analyses, tout ce qui relève des dynamismes d'ensemble au sein des sociétés serait négligé ou rendu négligeable; enfin, «notre dédain» pour les catégories réifiées ou édifiantes (la classe, la nation, le capitalisme, etc.) fait qu'il n'y aurait plus grandchose à comprendre de l'histoire, désormais réduite à un ensemble de flux plus ou moins saisissables et ne possédant d'autre matérialité que celle que l'historien veut bien lui donner par ses interprétations.

J'ignore si les Foucault, Geertz ou Darnton, trois auteurs cités au passage par le professeur et qu'il range du côté des culturalists, courant analytique qu'il abhorre, se reconnaîtraient dans la présentation impressionniste qu'il fait des thèses associées aux cultural studies, au linguistic turn et au poststructuralisme. J'en doute, car Foucault, Geertz et Darnton n'ont jamais tenu les positions radicales qui dérangent M. Nootens. On se demande d'ailleurs, parmi les chercheurs sérieux, qui a déjà défendu l'idée voulant que le monde n'existe que comme interprétation et discours? ou que l'acteur évolue indépendamment des systèmes englobants? ou que les individus et les groupes possèdent un noyau fondamental d'attributs et de caractéristiques? ou que le passé ne peut faire l'objet d'une étude rigoureuse, car l'histoire n'est toujours qu'un récit subjectif et relatif sur ce qui fut?

En fait, s'il est vrai de dire qu'au cours des trente dernières années, les perspectives microscopiques, l'anthropologie historique (à l'origine du relativisme interprétatif) et le "déconstructivisme» ont grandement inspiré les objets et méthodes de l'histoire, il est également juste d'affirmer que l'historiographie a été enrichie de ces études plutôt que dénaturée par elles. Les travaux de Carlo Ginsburg, d'Edward Thompson ou de Joan Scott, pour s'en tenir à ces auteurs, ont été complémentaires de ceux d'Immanuel Wallerstein, de Charles Tilly ou de Gérard Noiriel. Que dire par ailleurs des écrits de Paul Ricœur, de Marc Angenot ou de Jerome Bruner, ignorés par M. Nootens, mais qui ont énormément ajouté à notre compréhension des médiations narratives et discursives obligées par lesquelles les hommes entrent en rapport avec eux-mêmes et avec la société dans le temps?

Sous prétexte que les perspectives qu'il privilégie et que le type d'histoire qu'il pratique n'ont plus la cote qu'ils ont déjà eue, ce qui ne veut pas dire - heureusement d'ailleurs - qu'ils soient tombés en désuétude ou contestés dans leur pertinence, M. Nootens voudrait-il, en sévissant contre ce qui lui paraît «hors norme», "hors champ», ou "hors canon», se faire 
patrouilleur attitré du territoire de l'histoire et censeur patenté de la fonction d'historien? Pour ma part, j'ai toujours eu (sauf quand j'étais novice...) une vision très large de l'histoire, sur le plan des objets et des méthodes tout autant que sur celui des pratiques et des approches. La compréhension du monde, qui ne livre pas facilement ses secrets, nécessite à mon avis d'avancer sur plusieurs fronts à la fois de la connaissance. On me permettra de croire encore à la pluralité des visions et façons de faire (de) l'histoire.

Le point majeur de la critique du collègue consiste toutefois à dire que le concept d'identité, employé à toutes les sauces, est devenu insipide au point de fonder un nouvel essentialisme théorique. M. Nootens a peut-être raison de dire que certains auteurs se font champions d'un tel monisme. En ce qui me concerne, $\mathrm{j}$ 'ai toujours vu dans le concept d'identité un outil heuristique pour entrer dans la complexité du social et de l'individuel. Ce qui m'intéresse au fond, c'est de voir comment les sociétés, par ailleurs soumises au déchirement des conflits et à la dissonance des subjectivités et des relativismes de toutes natures, créent en leur sein, dirigées dans l'opération par des pouvoirs publics ou privés plus ou moins subtils, de la convergence, de la consonance et de la congruence. On est ici au cour de ce que certains appellent la régulation sociale, d'autres, la construction de l'hégémonie, d'autres encore la pratique de l'idéologie. Parler d'identité - et par conséquent d'identitaire, de grammaire identitaire, de construction identitaire, de politique identitaire, de référence identitaire, de patrimoine identitaire, etc. -, c'est étudier, selon les méthodes habituelles de l'histoire, comment les sociétés, pour exister matériellement sous la forme d'agrégats institués, doivent aussi produire du sens qui sécrète, en elles et chez leurs membres, de la dénomination commune, laquelle est aussi domination des uns par les autres.

Étudier l'identité d'une société, c'est en conséquence s'intéresser aux processus par lesquels cette société est amenée à se représenter comme un tout uni dans le temps et l'espace à travers - notamment - la construction de lieux de mémoire et l'élaboration d'une histoire qui octroient à cette société de la "substance», alors même que sa trajectoire est largement faite de contingences, de circonstances, d'incidences, de coïncidences et de chances. Étudier l'identité d'une société, c'est voir au fond comment cette société s'institue sur un plan également symbolique à travers la construction 
d'une référence qui, pour ses membres, crée de l'adhésion, de la participation, de l'identification et de l'illusion communautaire.

Or, ce qui se vérifie à l'échelle des collectivités se retrouve aussi chez les individus, mais différemment. Sans entrer dans la complication des choses ou prétendre fermer la question, disons que l'identité individuelle rend compte du processus par lequel un être humain développe sa subjectivité en se dotant notamment d'une histoire de lui-même par laquelle il contient ou compense sa tendance objective à la dispersion, se recentre autour d'une structure induisant un effet de continuité de/chez sa personne et tisse ses liens avec les autres, puisque l'histoire de soi fait toujours état de coempêtrements narratifs avec les histoires d'autrui. En avançant pareil point de vue, il ne s'agit pas de réduire l'acteur à une structure narrative, mais de soutenir que de vivre l'expérience humaine, c'est construire sur un mode réflexif, dialogique et narratif le sens diachronique et spatialisé de ses interactions sociales. (On m'excusera de stopper ici l'exposé de ma position, faute de place ${ }^{9}$.)

Pour conclure cette section, je me permettrai de réagir en rafale à certaines affirmations de M. Nootens, afin de le rassurer ou de nuancer son dire qui paraît souvent gonflé ou emporté :

- telle que je la conçois du moins, l'«approche identitaire» n'englobe pas tout et ne permet pas de saisir toutes les facettes d'une société ou d'un individu;

- pas plus que la classe ou la nation, deux concepts apparemment adulés par le collègue, l'identité (ou l'identitaire) ne constituent un explicateur universel de dernière instance;

- on ne peut déporter mécaniquement le concept d'identité dans des espaces-temps différents du nôtre non plus que coloniser le passé à partir de positions présentistes sur l'identitaire;

- enfin, j'ignore comment $\mathrm{M}$. Nootens parvient à la conclusion que la "posture identitaire va de pair avec la relégation, aux marges du récit historique, des tensions et rapports de pouvoir». Habituellement, ce sont les idéologues, parfois les journalistes, qui professent pareille satire. Ceux-là savent en effet que pour déprécier une problématique ou une perspective (on se souviendra de ce qui a été écrit sur les postulats du nouveau programme d'histoire), il faut la maculer au point de la rendre méconnaissable.

9. Pour plus de détails, voir Jocelyn Létourneau, «Clio en crise? Trois défis à l’interrogation historienne contemporaine», Edad media. Revista de Historia, 9 (2008): 87-103. 
J'en viens au dernier point de ma réplique.

Au début de son texte, $M$. Nootens se fait particulièrement dur contre moi en laissant entendre que ma véritable profession est celle d'éditorialiste plutôt que celle d'historien. Il s'en prend notamment à mon Passer à l'avenir, ouvrage qui a eu de l'écho et qui a nourri un débat important, loin d'être épuisé, sur l'avenir de l'histoire et de la mémoire au Québec. Le professeur allègue beaucoup de choses à mon endroit, mais prétend notamment que je serais partisan d'une instrumentalisation de l'histoire au bénéfice de créer à tout prix du vivre-ensemble au sein de la société (québécoise), ce qui fait que je poursuivrais l’objectif de «déconflictualiser» le passé, de mettre sous le tapis tout ce qui, dans ce passé, est susceptible de nuire au bonheur des contemporains, et tutti quanti.

Je vais être lapidaire ici: dans mes écrits, M. Nottens choisit de mettre en exergue ce qui fait son affaire pour construire son point de vue et discréditer mes positions, qu'il sort de leur contexte argumentatif ou simplifie abusivement. Compte tenu de l'espace qui m'est imparti, je ne peux évidemment repousser tous ses abordages non plus que résumer ce que j’ai publié depuis un bon moment sur la question de l'écriture de l'histoire. Une chose est sûre: l'idée d'enjoliver le passé pour permettre aux contemporains de passer à l'avenir n'est pas une idée que je soutiens. J'ai d'ailleurs été explicite à ce sujet dans de nombreux textes ${ }^{10}$. Ce que je crois par contre, c'est qu'il est plusieurs mises en récit valables du passé. D’où la question: dans le contexte d'une pluralité de narrations possibles de ce qui fut, quelle histoire offrir de l'ayant-été? Je prétends que le problème de la responsabilité de l'historien se profile dès l'instant où ce dernier conçoit son objet d'étude, le construit et exerce donc son métier d'interprétant.

Pour M. Nootens, ce genre d'interrogation et de préoccupation semble superflu. Il raisonne comme si le passé imposait sa propre narration objective que l'historien n'avait qu'à coucher subjectivement sur papier. Bien sûr, le collègue a le droit de prôner pareille conception mécanique de la discipline et de percevoir l'historien comme un fonctionnaire. J'envisage les choses sur un mode plus compliqué et astreignant en suggérant que l'historien, dans son récit d'histoire, doit faire avec la contrainte de rigueur (la méthode) et celle de pertinence (la mémoire ou le politique). J'en conviens, la contrainte de pertinence est délicate et incommode à négo-

10. Ibid. Voir aussi Jocelyn Létourneau, «Les possibilités de la narration historienne», dans Serge Jaumain et Éric Renacle, dir., Mémoire de guerre et construction de la paix. Mentalités et choix politiques, Belgique, Europe, Canada (Bruxelles, Peter Lang, 2006), 29-37. 
cier. Mais elle est incontournable, sauf pour ceux qui préfèrent l'éviter par pleutrerie ou démission intellectuelle.

À première vue, on pourrait penser qu'il existe une opposition, voire une contradiction, entre la contrainte de rigueur et la contrainte de pertinence. Je soutiens que le récit le plus juste de ce qui fut est aussi celui qui est le plus rigoureux, c'est-à-dire celui qui évite toute téléologie, qui refuse toute dichotomie, qui joue dans les zones d'ombre, qui établit l'ambiguité des situations historiques, qui insiste sur la polyvalence des enjeux, qui admet que l'action des hommes est pleine de contresens, d'équivoques, d'ambivalences, de confusions, de labilités, de flexions et de permutations. Or, ces lieux flous et incertains de l'action humaine, ceux où une chose et son contraire cohabitent de façon parfois surprenante, peuvent, s'ils sont investigués à des fins de construction historiale, être les nouds d'un récit différent du passé. Un récit qui montre à l'homme que son destin n'est attaché à aucune fin suprême ou supérieure ; qui lui rappelle à quel point il n'est déterminé par aucune force extérieure; qui lui dit combien il est libre de créer son histoire et donc de la changer aussi ; qui fait du gris, et non du noir ou du blanc, le lieu où se déroule le plus clair de l'action humaine; qui rappelle aux contemporains que le passé a toujours été un (mi)lieu de passage et de communication plutôt que d'impasse et de fermeture. Un récit, en définitive, qui pose l'espérance au cœur de la vie, y compris lorsqu'elle s'enfonce dans l'absurdité.

Que M. Nootens ne voie pas l'intérêt de s'interroger en ces termes ou qu'il règle la question de l'écriture de l'histoire en se rabattant sur le poncif voulant que l'historien, dans son travail professionnel, doive "s'en tenir à une application rigoureuse de ses méthodes d'enquête et de questionnement, cela en vue de rendre compte le mieux possible du passé », voilà qui est son affaire. Je ne prétends pas avoir trouvé la voie argumentative la plus lumineuse pour établir le rapport optimal entre la mémoire et la méthode. Je préfère toutefois attaquer le problème de front plutôt que de m'illusionner sur son inexistence en prétendant qu'il cache au fond une volonté de «triturer le passé». 